Optimizing ion-cyclotron resonance frequency heating for ITER: dedicated JET experiments

This article has been downloaded from IOPscience. Please scroll down to see the full text article.

2011 Plasma Phys. Control. Fusion 53124019

(http://iopscience.iop.org/0741-3335/53/12/124019)

View the table of contents for this issue, or go to the journal homepage for more

Download details:

IP Address: 194.81.223.66

The article was downloaded on 22/11/2011 at 11:00

Please note that terms and conditions apply. 


\title{
Optimizing ion-cyclotron resonance frequency heating for ITER: dedicated JET experiments
}

\author{
E Lerche $^{1}$, D Van Eester ${ }^{1}$, J Ongena ${ }^{1}$, M-L Mayoral ${ }^{2}$, M Laxaback ${ }^{3}$, \\ F Rimini ${ }^{2}$, A Argouarch ${ }^{4}$, P Beaumont ${ }^{2}$, T Blackman ${ }^{2}, \mathbf{V}$ Bobkov $^{5}$, \\ D Brennan ${ }^{2}$, A Brett ${ }^{2}$, G Calabro ${ }^{6}$, M Cecconello $^{7}$, I Coffey ${ }^{2}$, L Colas ${ }^{4}$, \\ A Coyne $^{2}$, K Crombe $^{8}$, A Czarnecka ${ }^{9}$, R Dumont $^{4}$, F Durodie ${ }^{1}$, \\ R Felton ${ }^{2}$, D Frigione ${ }^{6}$, M Gatu Johnson ${ }^{7}$, C Giroud ${ }^{2}$, G Gorini ${ }^{10}$, \\ M Graham ${ }^{2}$, C Hellesen ${ }^{7}$, T Hellsten ${ }^{3}$, S Huygen ${ }^{1}$, P Jacquet ${ }^{2}$, \\ T Johnson ${ }^{3}$, V Kiptily ${ }^{2}$, S Knipe ${ }^{2}$, A Krasilnikov ${ }^{11}$, P Lamalle $^{12}$, \\ M Lennholm ${ }^{2}$, A Loarte ${ }^{12}$, $\mathbf{R}$ Maggiora $^{13}$, M Maslov ${ }^{14}$, A Messiaen ${ }^{1}$, \\ D Milanesio $^{13}$, I Monakhov ${ }^{2}$, M Nightingale $^{2}$, C Noble $^{2}$, M Nocente $^{10}$, \\ L Pangioni ${ }^{2}$, I Proverbio ${ }^{10}$, C Sozzi $^{10}$, M Stamp $^{2}$, W Studholme ${ }^{2}$, \\ M Tardocchi $^{10}$, T W Versloot ${ }^{15}$, V Vdovin ${ }^{16}$, M Vrancken $^{1}$, \\ A Whitehurst $^{2}$, E Wooldridge $^{2}, \mathrm{~V}$ Zoita $^{17}$ and JET EFDA Contributors ${ }^{18}$ \\ JET-EFDA Culham Science Centre, Abingdon, OX14 3DB, UK \\ ${ }^{1}$ LPP-ERM/KMS, Association Euratom- 'Belgian State', TEC Partner, Brussels, Belgium \\ 2 EURATOM-CCFE Fusion Association, Culham Science Centre, UK \\ ${ }^{3}$ Fusion Plasma Physics, Association EURATOM-VR, KTH, Stockholm, Sweden \\ ${ }^{4}$ CEA (IRFM)-EURATOM Association, Saint-Paul-lez-Durance, France \\ 5 IPP (MPI)-EURATOM Association, Garching, Germany \\ ${ }^{6}$ C R Frascati, EURATOM-ENEA sulla Fusione, Frascati, Italy \\ ${ }^{7}$ Uppsala University, Association EURATOM-VR, Uppsala, Sweden \\ ${ }^{8}$ Department of Applied Physics, Ghent University, B-9000 Ghent, Belgium \\ ${ }^{9}$ Institute of Plasma Physics and Laser Microfusion, Warsaw, Poland \\ ${ }^{10}$ Instituto di Fisica del Plasma, EURATOM-ENEA-CNR Association, Milan, Italy \\ 11 SRC RF Troitsk Institute for Innovating and Fusion Research, Troitsk, Russia \\ 12 ITER Organization, Saint-Paul-lez-Durance, France \\ 13 Politecnico di Torino, EURATOM-ENEA sulla Fusione, Torino, Italy \\ ${ }^{14}$ CRPP-EPFL, Association EURATOM-Confédération Suisse, CH-1015 Lausanne, Switzerland \\ 15 FOM Institute ijnhuizen, Association EURATOM-FOM, Nieuwegein, the Netherlands \\ 16 RNC Kurchatov Institute, Nuclear Fusion Institute, Moscow, Russia \\ ${ }^{17}$ National Institute for Plasma Physics, Association EURATOM-MEdC, Bucharest, Romania
}

Received 4 August 2011, in final form 30 August 2011

Published 14 November 2011

Online at stacks.iop.org/PPCF/53/124019

\begin{abstract}
In the past years, one of the focal points of the JET experimental programme was on ion-cyclotron resonance heating (ICRH) studies in view of the design and exploitation of the ICRH system being developed for ITER. In this brief review, some of the main achievements obtained in JET in this field during the last 5 years will be summarized. The results reported here include important
\end{abstract}

\footnotetext{
${ }^{18}$ See the appendix of Romanelli F et al 2010 Proc. 23rd IAEA Fusion Energy Conf. 2010 (Daejeon, Korea).
} 
aspects of a more engineering nature, such as (i) the appropriate design of the RF feeding circuits for optimal load resilient operation and (ii) the test of a compact high-power density antenna array, as well as RF physics oriented studies aiming at refining the numerical models used for predicting the performance of the ICRH system in ITER. The latter include (i) experiments designed for improving the modelling of the antenna coupling resistance under various plasma conditions and (ii) the assessment of the heating performance of ICRH scenarios to be used in the non-active operation phase of ITER.

(Some figures in this article are in colour only in the electronic version)

\section{Introduction}

Ion-cyclotron resonance frequency (ICRF) heating is one of the main auxiliary heating systems foreseen for ITER [1,2]. Together with neutral beam injection (NBI, $33 \mathrm{MW}$ ) and electroncyclotron resonance heating (ECRH, 20 MW), it is expected to provide $20 \mathrm{MW}$ of heating power to help the ITER plasmas in achieving fusion relevant temperatures. Because most of the ICRF power applied is typically absorbed by the ions, this heating method is expected to have the strongest impact on the DT fusion yield per MW of external power applied to the plasma.

The basic principles of ICRF heating are illustrated in figure 1:

(1) A high-power radio-frequency (RF) generator is connected by a long transmission line to poloidal strap antennas located inside the vacuum vessel of the tokamak. A matching circuit (composed of transmission line elements of variable length) is used to ensure realtime impedance matching between the high- $Z$ generator and the antenna array during a plasma discharge.

(2) The antenna excites fast wave modes, which are evanescent in the low-density region of the scrape-off layer (SOL) but become propagative near the plasma separatrix, where the plasma density becomes larger.

(3) The fast wave modes propagate towards the plasma centre and transfer their energy to the plasma particles both by non-well-localized absorption processes, such as electron Landau damping (ELD) and transit time magnetic pumping (TTMP), and by well-localized resonant ion-cyclotron absorption, when the waves encounter the cyclotron layer of a given ion species, for which the wave frequency $\omega$ is equal to (or a multiple of) the local ioncyclotron frequency $\omega_{\mathrm{ci}}=q_{\mathrm{i}} / m_{\mathrm{i}} \cdot B_{0}$. The resonant ions, which are typically accelerated to high energies, transfer the absorbed wave power to the bulk plasma via collisions (slowing-down) resulting in efficient plasma heating.

For the full process to be efficient, each of the above-mentioned steps has to be optimally accomplished:

(1) The generated RF power has to be properly transferred to the antenna, which depends on the antenna design and on the layout of the impedance matching circuit, which should be capable of maintaining the power reflected from the antenna below a certain level independent of the value of the antenna loading resistance. The quality of impedance matching is usually expressed in terms of the voltage standing wave ratio VSWR = $\left(\left|V_{\mathrm{FOR}}\right|+\left|V_{\mathrm{REF}}\right|\right) /\left(\left|V_{\mathrm{FOR}}\right|-\left|V_{\mathrm{REF}}\right|\right)$, where $V_{\mathrm{FOR}}$ and $V_{\mathrm{REF}}$ are, respectively, the forward and reflected voltages measured by directional couplers installed in the feeding transmission 


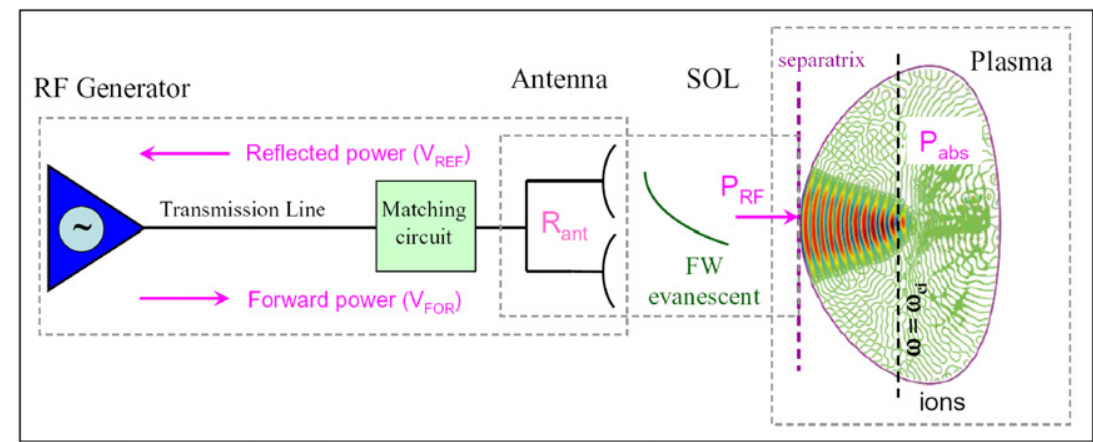

Figure 1. Chart illustrating the principle of the ICRF heating process.

line. VSWR is 1 when no power is reflected from the antenna (perfect match) and grows when the reflection is finite. If the VSWR value close to the RF generator is too high (the limit being imposed by the operator according to the RF settings of a given experiment), too large a fraction of the power comes back to the generator and may damage it; consequently, the generator is shut down for protection reasons.

(2) The power launched by the antenna has to be efficiently coupled to the plasma, which also depends on the antenna design but is particularly sensitive to the characteristics of the plasma in the SOL. The efficiency of this process is described by the antenna coupling resistance $R_{\text {ant }}$, which is proportional to the Poynting flux of the RF fields excited and thus describes the amount of RF power that tunnels through the evanescence region and reaches the plasma for a given voltage $\mathrm{V}$ applied to the antenna, $P_{\mathrm{RF}} \propto R_{\mathrm{ant}} \cdot V^{2}$.

(3) The coupled RF power has to be efficiently absorbed in the plasma, which depends not only on the antenna excitation but also on the plasma core parameters $\left(n_{\mathrm{e}}, T_{\mathrm{e}}\right.$ and plasma composition) and on the equilibrium magnetic field $B_{0}$. The figure of merit of this process is the heating efficiency $\eta=P_{\mathrm{abs}} / P_{\mathrm{RF}}$, which quantifies the amount of power that is truly absorbed in the plasma $\left(P_{\mathrm{abs}}\right)$, as opposed to the power lost by edge processes and ICRF-induced radiation losses.

It is clear that maximizing the performance of each of the above-described steps is a big challenge, particularly for ITER where, on top of several design and engineering constraints, ICRF is supposed to reliably deliver a significant amount of power in different operation phases and in different heating scenarios, despite the unfavourable conditions of the SOL (large antenna-plasma distances, large edge density variations due to edge localized modes (ELM), etc).

This work summarizes the latest contributions of the JET experimental programme for enhanced ICRF operation under various conditions, aiming at optimizing the design and operation of the ICRF system for ITER. The paper is divided as follows: section 2 describes the modifications made in the matching circuit layout of the JET A2 antennas in order to allow efficient RF power coupling during the fast antenna loading variations caused by ELMs. Section 3 summarizes the key results obtained with the 'ITER-like' antenna (ILA), a new compact antenna array installed in JET with the objective of testing key features of the ITER antenna design; In section 4, the results of experiments designed to validate RF modelling tools and in particular coupling resistance calculations will be shown, including a review of the estimated coupling expected in ITER. In section 5, the results of some ICRF scenarios proposed for the non-active operation phase of ITER that were recently tested in JET will be 


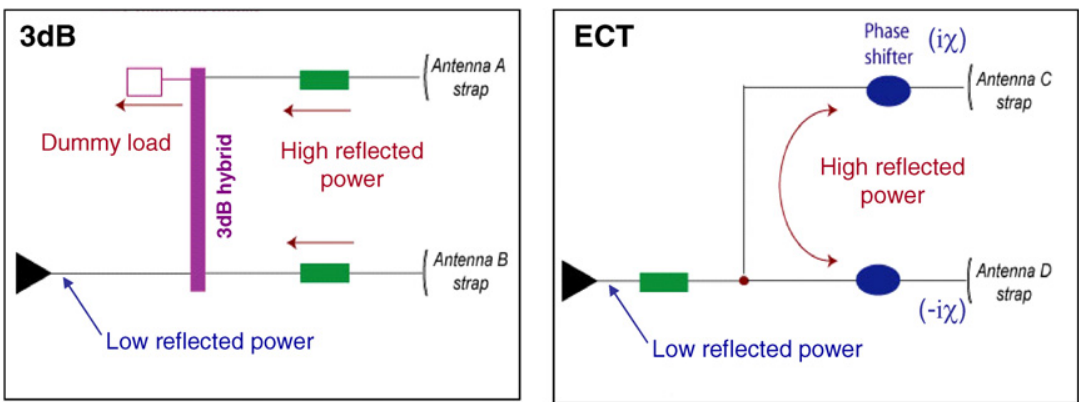

Figure 2. Load resilient circuit layouts of the A2 antennas: (left) the $3 \mathrm{~dB}$ hybrid concept used in the antenna modules A and B; (right) the (external) conjugate-T configuration (ECT) used to operate antennas C and D in ELM resilient mode.

summarized and preliminary predictions of their performance in ITER will be drawn. The paper ends with a brief summary and some plans for future investigations.

\section{ELM resilient operation of the JET A2 antennas}

An ion-cyclotron resonance heating (ICRH) system for ITER and fusion reactor applications should be able to cope with fast antenna load variations, such as those produced by ELMs or by pellet injection. In the A2 antenna system in JET [3], consisting of four antenna modules each composed of four straps fed individually by one amplifier, impedance matching is achieved with the use of mechanical transmission line elements (stubs and trombones), which are too slow to follow the fast (order $\sim 10-50 \mu \mathrm{s}$ ) antenna load variations induced by ELMs. This leads to large amounts of reflected power returning to the amplifier, which, to protect itself, shuts down for a certain interval of time. The average power delivered to the plasma is therefore much less than the requested one, with minimal plasma heating as a result.

To demonstrate a solution to this problem two different load resilience concepts have been implemented and tested at JET with the A2 antennas:

(i) A $3 \mathrm{~dB}$ hybrid matching circuit [4]: such a system is based on inductively connecting pairs of straps with $3 \mathrm{~dB}$ hybrid couplers and thereby diverting the reflected power occurring during loading transients to a dummy load (see figure 2(a)). This approach was first proven on ASDEX-Upgrade [5]. It provides safe operational conditions for the RF generators at the expense of wasting a fraction of the generated power during ELMs to the dummy load. This system is currently installed on two of the four A2 antenna modules (antennas A and B) and allowed trip-free ICRF operation during most types of ELMs, with timeaveraged power levels delivered to $\mathrm{H}$-mode plasmas up to three times larger than what was previously possible.

(ii) The conjugate- $\mathrm{T}$ concept (CT), involving pairs of straps connected in parallel to form so-called resonant double loops (RDLs) [6]. The two straps in each RDL are fed from a common transmission line via a T-junction, with adjustable matching elements (phase shifters) located between the antenna straps and the conjugate T-point (see figure $2(b)$ ). Tolerance to plasma load variations is achieved by adjusting the impedances of the two branches to be complex conjugate. Ideally, this results in a purely resistive load seen by the amplifier and as long as the load variations experienced by the two antenna straps are similar, the reflected power keeps being transferred from one antenna branch to the other (within the RDL circuit) resulting in low reflected power levels in the circuit behind the 


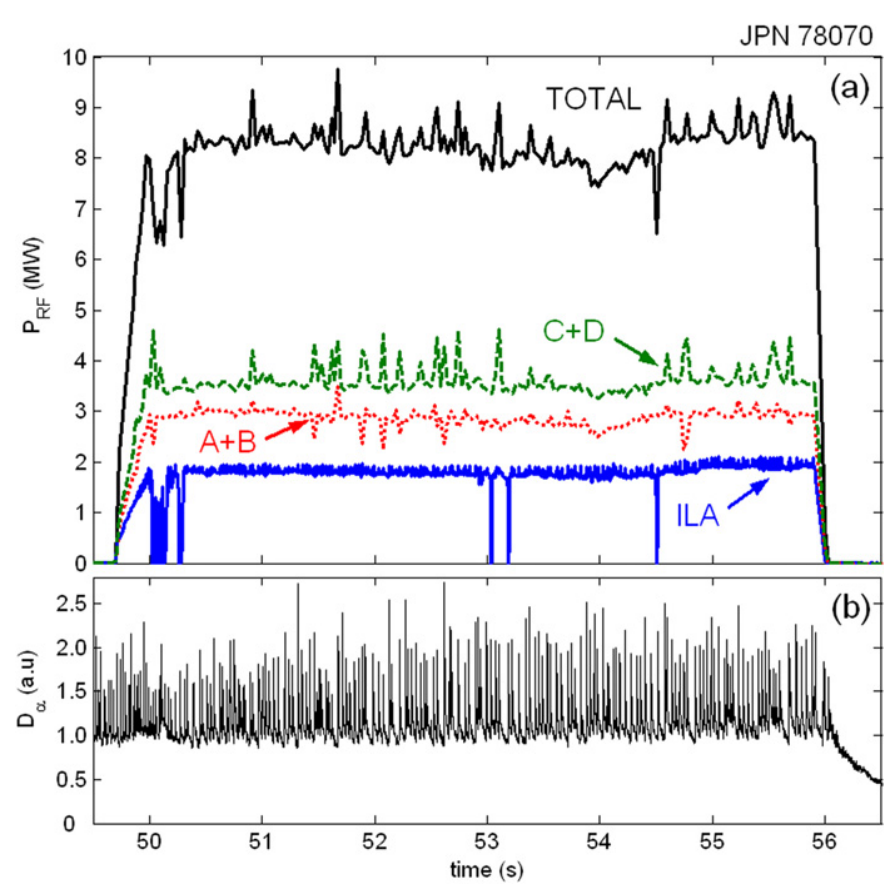

Figure 3. ELM resilient operation of the ICRF system in JET: (a) ICRF power coupled by antennas $\mathrm{A}+\mathrm{B}$ (dotted), antennas $\mathrm{C}+\mathrm{D}$ (dashed) and by the ILA (solid); $(b) \mathrm{D}_{\alpha}$-emission illustrating the strong type-I ELMs in the discharge.

conjugate T-point independent of the value of the loading resistance of the antenna. Such a system with line stretchers (external to the tokamak) as adjusting impedance elements is installed on antennas C and D [7] and also demonstrated good reliability for ICRF operation during strong ELMs. This approach has the advantage that the RF power is also coupled to the plasma during the ELMs (rather than being deviated to an external dummy load) but requires careful tuning of the individual antenna branches for achieving wide load tolerance under different plasma conditions, as opposite to the $3 \mathrm{~dB}$ concept which is based on a simpler matching principle.

Both the $3 \mathrm{~dB}$ and the conjugate-T configurations installed in the $\mathrm{A} 2$ antenna system showed good RF power coupling reliability in ELMy H-mode plasmas. A second ELM resilient system based on the conjugate-T principle is installed at JET on the ILA, as will be described in the next section. Together with the ILA, the ICRF system coupled more than $8 \mathrm{MW}$ in strong type-I ELMy H-mode discharges in JET [8]. An example of one of these pulses is given in figure 3, where the time traces of the ICRF power coupled by each of the load resilient systems (a) are shown together with the $\mathrm{D}_{\alpha}$-emission signal (b).

\section{Key results of the JET ILA}

The concept of high-power compact ICRF antennas was put to test with the installation in 2008 of the ILA, a compact antenna array $\left(0.9 \mathrm{~m}^{2}\right)$ composed of eight poloidal straps $[9,10]$. Each pair of straps is connected in the conjugate-T configuration for extended load tolerance and the individual branch reactances are tuned with internal matching capacitors. Apart from ELM resilience, this antenna has been specially designed to test key specifications of the ICRF 

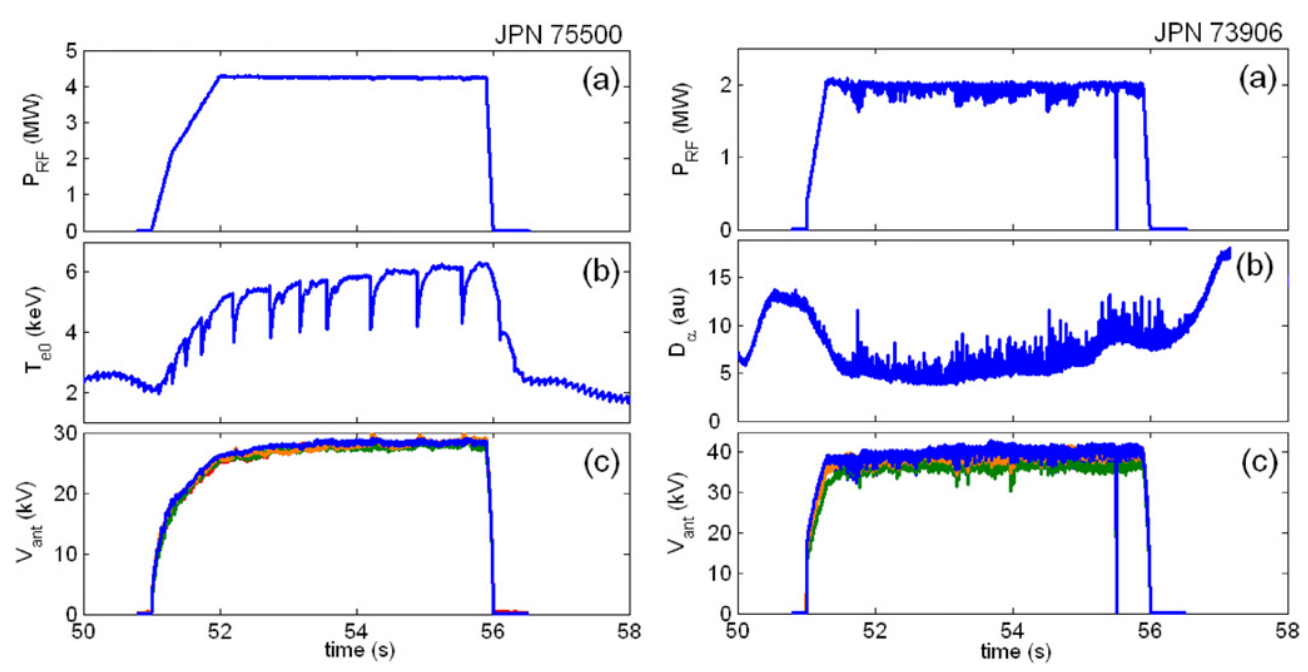

Figure 4. (left) High-power density ILA pulse (L-mode): (a) ICRF power, $(b)$ central electron temperature, $(c)$ voltage on four antenna straps; (right) high antenna voltage ILA pulse (H-mode): (a) ICRF power, (b) $\mathrm{D}_{\alpha}$ emission, (c) voltage on four antenna straps.

antenna proposed for ITER, requiring reliable operation at high strap voltages and at highpower densities with real-time matching of the full antenna array, in which the neighbouring straps are strongly coupled to each other.

In figure 4(left), an example of a high RF power density pulse is given. After the application of $4.3 \mathrm{MW}$ of ICRF power $\left(\sim 5 \mathrm{MW} \mathrm{m}^{-2}\right)$, the electron temperature increases by $3-4 \mathrm{keV}$ illustrating the fact that efficient plasma heating is taking place and that RF-induced radiation losses are small. The large sawtooth oscillations [11] observed in the $T_{\mathrm{e}}$ signal further corroborate the efficient $\mathrm{H}$ minority ion acceleration in the plasma core. This known effect is a consequence of the stabilization of the internal kink mode due to the presence of RF accelerated ions inside the $q=1$ surface, causing the sawtooth crashes to be less frequent and of a larger amplitude [12]. Also note that in this case, which features high coupling resistance, the strap voltages are only about $30 \mathrm{kV}$ and there is an almost perfect voltage balance on the several antenna straps (c). In similar pulses, a maximum power density of $6.2 \mathrm{MW} \mathrm{m}^{-2}$ is achieved, which corresponds to the ballpark of the values currently specified for ITER $\left(6-7 \mathrm{MW} \mathrm{m}^{-2}\right)$. In figure 4(right), an example of a high-voltage operation with the lower half of the ILA antenna is given. Because this is a $\mathrm{H}$-mode discharge, the coupling resistance is relatively low and for coupling about $2 \mathrm{MW}$ of RF power with half of the antenna it was necessary to increase the antenna voltages to $\sim 40 \mathrm{kV}$. Also note that the strap voltages are somewhat less balanced than in the L-mode discharge (left), a consequence of the enhanced influence of the mutual coupling between straps on the antenna control when the coupling resistance is low [13]. The maximum voltage achieved in arc-free ILA operation was about $43 \mathrm{kV}$, a value that again meets the requirements proposed for the ITER antenna $(V<45 \mathrm{kV})$.

The good performance of the ILA antenna in JET together with extensive RF modelling of the experimental results increased the confidence on key aspects of the ITER antenna design, in particular on the capability of operating compact antennas with high-power densities and at high strap voltages. Unfortunately, due to a technical failure of one of the matching capacitors, only half of the antenna array remained operational during most of the H-mode commissioning phase and other important milestones, such as demonstrating full power capabilities in ELMy H-mode plasmas, could not be achieved. 

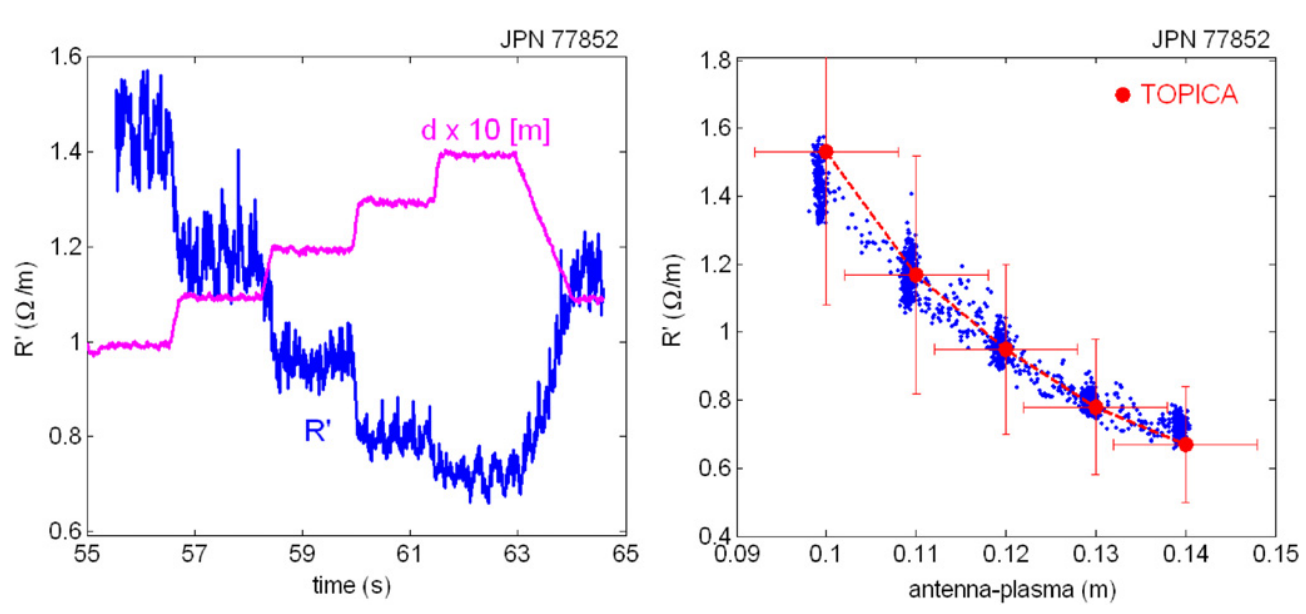

Figure 5. (left) Time traces of the coupling resistance of one pair of the ILA straps together with the antenna-plasma distance $(\times 10)$ in L-mode pulse 77852 at $f=42 \mathrm{MHz}$; (right) coupling resistance versus antenna-plasma distance for the same discharge together with the numerical modelling done with the TOPICA code.

\section{Antenna coupling studies}

As mentioned, the coupling resistance (or loading resistance) of the antenna is proportional to the Poynting flux associated with the RF fields near the plasma edge and therefore 'dictates' the RF power that can be coupled to the plasma for a given voltage or current in the antenna straps. This quantity not only depends on several RF parameters, such as the wave frequency and the $k_{\|}$spectrum excited by the antenna, but is particularly sensitive to the properties of the SOL and the plasma edge. To illustrate the parametric dependence of the coupling resistance with a few key quantities and to benchmark the numerical modelling tools used for designing the ITER ICRF antenna, a series of experiments was carried out in which the antenna-plasma distance was scanned during each pulse while the loading resistance was monitored, as illustrated in figure 5(left). In figure 5(right), the coupling resistance of one pair of the ILA straps is plotted against the antenna-plasma distance for an L-mode discharge at $f=42 \mathrm{MHz}$. As expected, the resistance decreases exponentially with the antenna-plasma distance [14] and it varies by a factor of $\sim 2$ when the plasma is shifted away from the antenna by $4 \mathrm{~cm}$. The modelling done with the TOPICA code [15] confirms this behaviour and is in very good agreement with the experimental data. The large error bars associated with the numerical predictions are a consequence of the uncertainties in the RF measurements and in particular in the edge density measurements (exact location of the cut-off position).

In figure 6(left), the coupling resistances obtained in two similar L-mode discharges operating at $42 \mathrm{MHz}$ and $33 \mathrm{MHz}$ are compared. It is clear that the coupling resistance values (and thus the power capabilities) are reduced when operating at lower frequencies, because these waves are more evanescent than the high-frequency waves in the SOL leading to smaller RF fields in the plasma for the same voltage imposed in the antenna. Figure 6(right) illustrates the effect of the density gradient in the plasma edge on the antenna loading, where the reference L-mode discharge at $f=42 \mathrm{MHz}$ shown in figure 5 is compared with an H-mode discharge with the same RF operating frequency. One sees that the coupling resistance is reduced for the case of the H-mode discharge and that this reduction is even stronger when the antennaplasma distance is smaller. The main reason for the $R_{\text {ant }}$ decrease in H-mode is not the change 

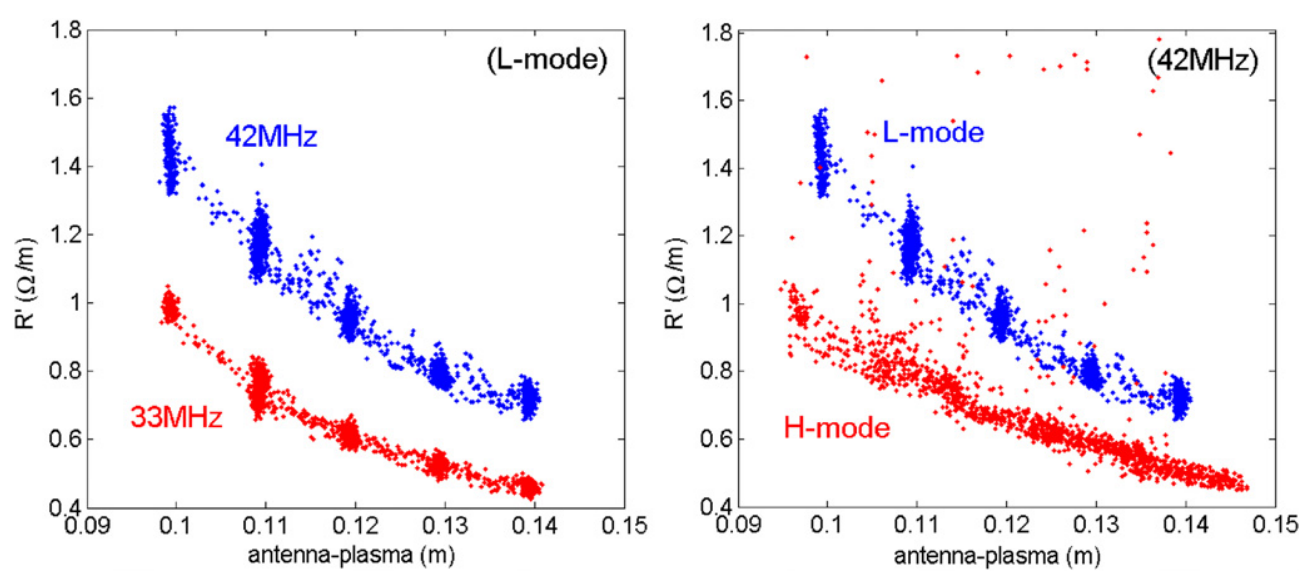

Figure 6. (left) Comparison of the coupling resistance obtained in the reference pulse 77852 (Lmode, $42 \mathrm{MHz}$ ) with a similar discharge with the RF operating frequency reduced to $f=33 \mathrm{MHz}$ (JPN 77847); (right) comparison of the reference pulse with a discharge with the same operating frequency but in H-mode (JPN 77851), where steeper density gradients are present in the plasma edge.
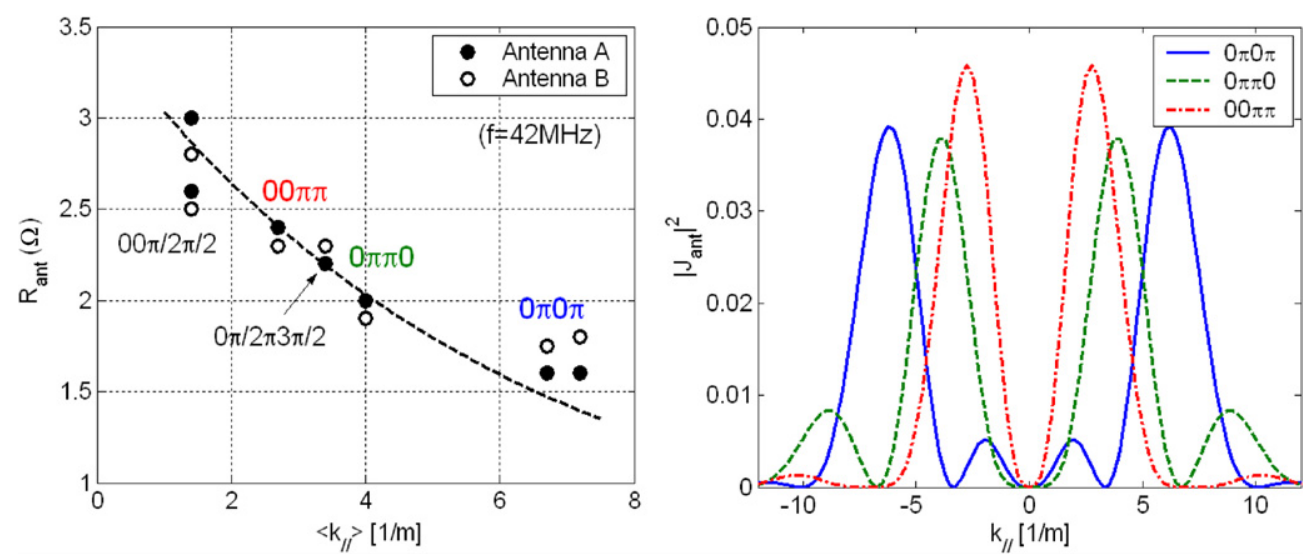

Figure 7. (left) Coupling resistance of the $\mathrm{A} 2$ antennas as a function of the dominant $k_{\|}$wavenumber excited in different phasing configurations (JPN 74091-74094, 78727-78732); (right) example of the $k_{\|}$-spectra excited by the A2 antennas computed with the ANTITER II code for three cases: $0 \pi 0 \pi$ (solid), $0 \pi \pi 0$ (dashed) and $00 \pi \pi$ (dashed-dotted).

in the SOL density values but the steeper density gradients that exist in the plasma edge (inside the propagative region of the waves) in the H-mode discharges, which are characterized by a higher pedestal density. This effect is called refractive index mismatch and is related to the higher wave reflection in the case of steep refractive index (density) gradients [16].

In a different series of experiments done with the A2 antennas, the influence of antenna phasing on the coupling resistance was investigated. In figure 7 the average coupling resistances of the antenna modules $\mathrm{A}$ and $\mathrm{B}$ are plotted as a function of the dominant $k_{\|}$wavenumber excited in each of the different antenna phasings adopted. In figure 7(right) the corresponding $k_{\|}$-spectra excited in three phasing configurations computed with the ANTITER II code [17] are illustrated: $0 \pi 0 \pi$ (solid), $0 \pi \pi 0$ (dashed) and $00 \pi \pi$ (dashed-dotted). All the pulses were 
done at $B_{0}=3.0 \mathrm{~T} / I_{\mathrm{P}}=2 \mathrm{MA}$ with an RF frequency of $f=42 \mathrm{MHz}$ and a fixed antennaplasma distance of $d=0.11 \mathrm{~m}$. The dashed curve on the left figure illustrates the approximate exponential dependence of the coupling resistance with the dominant $k_{\|}$wavenumber excited. Note that by operating at, e.g., $00 \pi \pi$, the coupling resistance can be improved by about $30 \%$ with respect to the standard dipole configuration $(0 \pi 0 \pi)$ typically used.

In this same experimental campaign, the ICRF heating efficiency as well as the RF-induced plasma-wall interaction was also studied. It was observed that, for the $T=5 \mathrm{keV}$ target plasmas adopted in these experiments, operating at antenna phasings with dominant $k_{\|}$ values lower than $4 \mathrm{~m}^{-1}$ caused a strong degradation in the heating performance [18]. The decrease in the plasma absorptivity at low $k_{\|}$has been corroborated numerically by 1D wave calculations done with the TOMCAT code [19] and is related to the narrower ioncyclotron absorption region obtained at low $k_{\|}$for a given plasma temperature. Preliminary predictions indicate that this deleterious effect will be negligible in ITER even when operating at the lowest $k_{\|}$phasing foreseen $(00 \pi \pi)$, as long as the plasma temperature is sufficiently high $(T>8-10 \mathrm{keV})$. Another important outcome of these experiments was the enhanced plasma-wall interaction observed at low $k_{\|}$phasing configurations, leading to a higher plasma impurity content and therefore higher RF-induced radiation losses [20,21]. These observations are consistent with enhanced RF sheath rectification effects, i.e. with the fact that the parallel RF electric field excited close to the antenna is larger for the low $k_{\|}$phasing configurations (where neighbouring pairs of straps are fed with currents of equal phase) and non-resonant acceleration of charged particles in the SOL is enhanced [22]. Numerical modelling of these experiments using the HFSS code confirms that the near fields and the image currents excited in the antenna box are indeed expected to be larger in the low- $k_{\|}$ cases [23].

As mentioned before, the value of the plasma density in the SOL and in the vicinity of the cut-off density region also has a strong influence on the coupling resistance. Experiments aiming at enhancing the antenna loading by injecting small amount of gas from different locations in the tokamak under conditions similar to those expected in ITER were carried out on JET and on other machines [24, 25]. Although gas injection from the divertor, top or midplane, led to a global modification of the SOL density profiles significant enough to improve the ICRF coupling, it was also shown that an injection near the antennas could lead to an additional ICRF coupling improvement for the same amount of gas injected. As expected, gas injection was found to affect (differently depending on the plasma configuration pumping and recycling properties) the plasma pedestal and hence the bulk plasma confinement properties, but so far the disadvantages associated with such a decrease compared with the advantages of a potential increase in the power input to the plasmas centre have not yet been quantified [24].

The strong sensitivity to the SOL properties is further reflected in the numerical predictions of the power capabilities of the ITER ICRF antennas. Recent studies have shown that the expected coupling resistance can change by a factor of roughly 6 when considering the most optimistic and the most pessimistic SOL density profiles currently computed for ITER [17]. Even in the worst case scenario (in which the density is so low that one could claim that the plasma can safely be shifted closer to the antenna to enhance the coupling) the power coupled per antenna module is about $12 \mathrm{MW}$, still above the minimum specifications of the ITER antenna design (10 MW). In the same study it was also shown that, by operating at the standard dipole antenna phasing configuration $(0 \pi 0 \pi)$ instead of operating with the reference phasing $0 \pi \pi 0$, the coupling resistance can be degraded by a factor of 2 . Efforts trying to reduce the uncertainties on the ICRF power capability predictions for ITER based on adopting different SOL profiles, which nevertheless feature similar heat loads in the first wall (rather than similar antenna-plasma distances), are ongoing. 

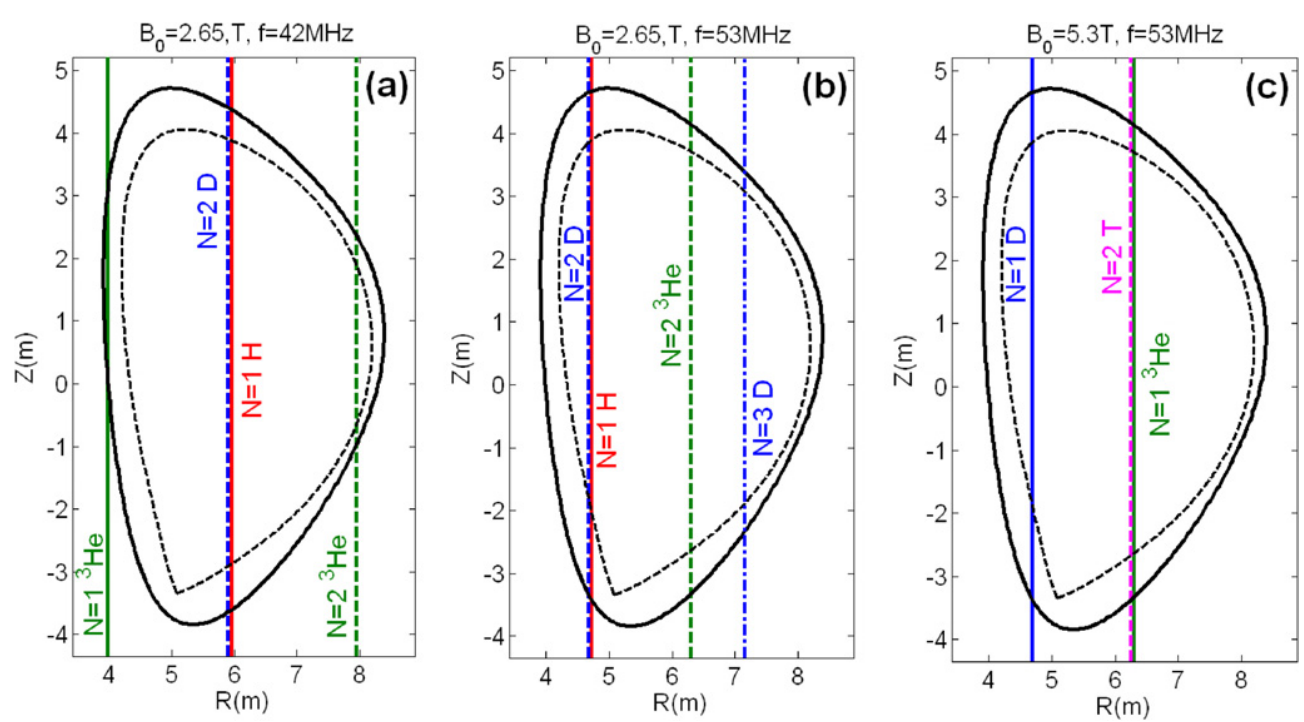

Figure 8. Location of the main fundamental (solid), 2nd harmonic (dashed) and 3rd harmonic (dashed-dotted) ion-cyclotron resonances of various ion species under different conditions for ITER: $(a) B_{0}=2.65 \mathrm{~T}$ and $f=42 \mathrm{MHz} ;(b) B_{0}=2.65 \mathrm{~T}$ and $f=53 \mathrm{MHz} ;(c)$ full-field DT operation at $B_{0}=5.3 \mathrm{~T}$ and $f=53 \mathrm{MHz}$.

\section{Development of ICRF heating schemes for ITER's non-active phase}

Prior to the main phase of exploitation to be performed at the full magnetic field of $5.3 \mathrm{~T}$ with deuterium-tritium plasmas [26,27], the ITER tokamak will first go through a commissioning phase at half of its nominal magnetic field $\left(B_{0}=2.65 \mathrm{~T}\right)$. This phase is intended to test the plasma behaviour and its main properties while full heating becomes available and to test the diagnostics in a less harsh environment than that of a burning plasma. To avoid premature activation of the machine, hydrogen $(\mathrm{H})$ and/or helium $\left({ }^{4} \mathrm{He}\right)$ rather than deuterium (D) and tritium (T) will be adopted as a majority gas during that initial phase.

In the foreseen frequency range of the ITER ICRF system ( $f=40-55 \mathrm{MHz})$ the following heating scenarios are possible for ITER's non-activated phase at half-field:

(i) Hydrogen heating at its fundamental $(N=1)$ cyclotron resonance around $f=42 \mathrm{MHz}$ (see figure $8(a)$ ), either in ${ }^{4} \mathrm{He}$ plasmas or in pure $\mathrm{H}$ plasmas. While the latter (known as $\mathrm{H}$ minority ICRF heating) is commonly used in present-day tokamaks due to its high heating efficiency, the ion-cyclotron heating of single ion species plasmas is known to be less efficient [28] and therefore has not been as much explored in current day machines.

(ii) ${ }^{3} \mathrm{He}$ heating at its second harmonic $(N=2)$ cyclotron resonance around $f=53 \mathrm{MHz}$ in $\mathrm{H}$ or ${ }^{4} \mathrm{He}$ plasmas (see figure $8(b)$ ). In view of the sizeable volume of ITER and the cost of this helium isotope, large ${ }^{3} \mathrm{He}$ concentrations will however be avoided in this next-step machine. This compromises the efficiency of this heating scenario, as will be shown later. Note that this scheme is a mock-up of the heating scheme foreseen for the machine's activated DT phase: as the charge-to-mass ratio $(Z / A)$ of $\mathrm{D}$ and $\mathrm{T}$ is half of the ratio for $\mathrm{H}$ and ${ }^{3} \mathrm{He}$, the respective cyclotron layers in $\mathrm{H}-{ }^{3} \mathrm{He}$ plasmas at half field are at the same positions as the D-T cyclotron layers at ITER's full field, $B_{0}=5.3 \mathrm{~T}$ (see figure $8(c)$ ). Therefore, the local wave dispersion and thus the RF field structure will be similar in both cases. 

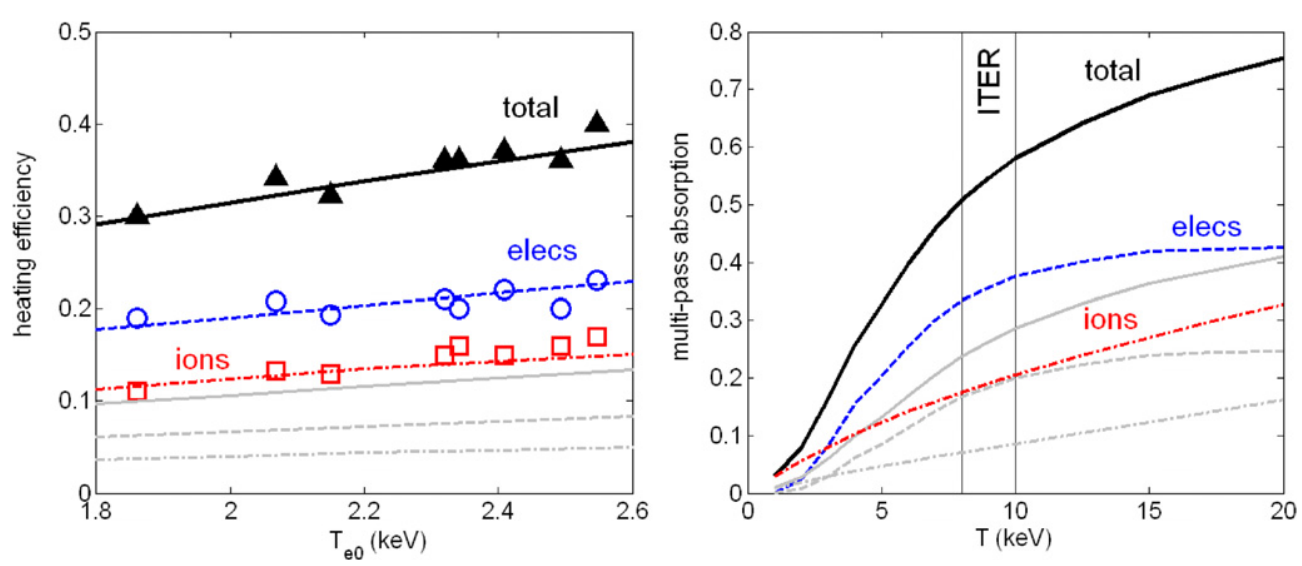

Figure 9. (left) Experimental heating efficiencies (ions-squares, electrons-circles, totaltriangles) obtained in the $\mathrm{H}$ majority heating experiments in JET (JPN 79330-79335) as a function of the plasma temperature together with the multi-pass absorption predictions (ions-dasheddotted, electrons - dashed, total-solid) based on the single pass absorption values computed with the TOMCAT code (light grey curves) by considering $22 \%$ of power losses per wave pass in the plasma; (right) multi-pass absorption (ions — dashed-dotted, electrons-dashed, total—solid) estimated from the TOMCAT results for ITER's half-field plasma conditions adopting the same losses as found from the JET experiments (again, the grey curves indicate the single pass absorption values used in the multi-pass model).

In preparation for ITER's half-field operation phase, and as the JET A2 frequency range allows it, a set of experiments was conducted in $\mathrm{H}$ plasmas to test the two heating schemes just described [29]. The RF frequency used was $f=42 \mathrm{MHz}$ for the fundamental $\mathrm{H}$ majority heating experiments and $f=52 \mathrm{MHz}$ for the $N=2{ }^{3} \mathrm{He}$ ones. Dipole phasing was adopted and up to $P_{\mathrm{RF}}=5.5 \mathrm{MW}$ was coupled to the plasma. The central density was around (3.0-3.5) $\times 10^{19} \mathrm{~m}^{-3}$, which is comparable to the density expected in the initial operation of ITER. Central ion and electron temperatures of $2-3 \mathrm{keV}$ were reached, which are well below the expected temperatures of the initial ITER plasmas $(8-10 \mathrm{keV})$.

Figure 9(left) depicts the measured (symbols) and calculated (lines) heating efficiencies for the $\mathrm{H}$ majority heating scenario as a function of the plasma temperature. The experimental data were obtained by modulating the RF power and studying the ion and electron temperature responses by break-in-slope analysis techniques [30]. JET is equipped with diagnostics that allow pinning down the temperature profiles with sufficient spatial and temporal resolution to yield meaningful results about where the externally launched waves transfer their energy to the plasma. The ion temperature profile is obtained via charge exchange diagnostics [31], while the electron temperature profile is estimated from electron cyclotron emission measurements [32].

One can clearly see that the electron absorption $(\sim 20 \%)$ systematically dominates the ion absorption $(\sim 15 \%)$ and that the total heating efficiency slightly increases with the plasma temperature from $30 \%$ to $40 \%$ in the studied interval. These low values indicate that this is not a particularly efficient heating scheme (a typical figure of merit for hydrogen minority heating in D plasmas - the most widely used RF heating scheme in JET-is around 80-90\%). A nonnegligible fraction of the power is lost through RF-induced radiation $(\sim 30 \%)$, and the scaling of the impurity content with the RF power suggests that there is significant interaction of the edge electric fields with the wall. Note that up to a point, optimizing the antenna structure allows reducing the sheath effects near the antenna but the large electric fields set up at the plasma edge and in the SOL are hard to avoid in schemes characterized by low single pass absorption. 
The importance of taking into account the RF power losses in low absorption scenarios can readily be seen when comparing the theoretical single pass absorption predictions obtained by the 1D wave code TOMCAT [19] (grey curves in figure 9, left) with the experimental findings: although the single pass absorption values confirm the relative importance of ion versus electron heating, the summed absorption is very low $(\sim 10 \%)$. When realizing that the power is sloshing through the machine a number of times before being fully absorbed by the plasma, it becomes clear that a model that includes the edge losses per single pass of the ICRF waves in the plasma is needed to simulate the experimental heating efficiencies found. As shown in figure 9(left), good agreement between the experimental values and the multi-pass absorption predicted by TOMCAT is obtained when single pass losses of $22 \%$ are considered in the multi-pass model, a value that is twice as large as the single pass absorption $(\sim 10-12 \%)$.

When examining the same scenario for ITER (figure 9, right), similar conclusions as for JET can be drawn, although the size of the machine and the higher temperatures yield somewhat less pessimistic single pass absorption figures: at the temperature projected to be reached in the L-mode ITER phase $(8-10 \mathrm{keV})$ the ions absorb $7 \%$ of the power while the electrons absorb $18 \%$ in a single transit over the plasma, yielding a global single pass absorption in the range of $25 \%$ (grey curves). Assuming that the losses will be similar in the edge of the ITER machine as those found for JET, the projected overall heating efficiency is in the range $50-60 \%$ in the temperature range expected in ITER. The fact that the obtained figure sensitively depends on the plasma temperature underlines the need for self-consistent modelling of the heating and transport, a topic outside the scope of this paper. Apart from more in depth theoretical analysis to optimize the performance of this ICRF scenario, further experimental study can help to improve this heating scheme in preparation for ITER. Theoretical and experimental evidence suggests that, provided efficient means of preheating the plasma are available, this scheme's efficiency may be increased. Another modestly promising option that has been identified is the change of the gas mixture (e.g. dilution with ${ }^{4} \mathrm{He}$ ) to profit from the beneficial role of the change of the RF wave polarization on the heating efficiency.

Except for the different RF frequency and the presence of ${ }^{3} \mathrm{He}$ in the discharges, the plasma parameters for the second harmonic ${ }^{3} \mathrm{He}$ ICRF experiments were the same as those used for the fundamental $\mathrm{H}$ heating experiments. The ${ }^{3} \mathrm{He}$ content was scanned from $\mathrm{X}\left[{ }^{3} \mathrm{He}\right]=2 \%$ to $25 \%$ and was controlled in real time by an algorithm that estimates the amount of ${ }^{3} \mathrm{He}$ present in the discharge based on edge spectroscopy measurements [33]. Similar to the above discussed $\mathrm{H}$ majority case, this scenario proved to be a heating scheme of modest potential, in particular at the low ${ }^{3} \mathrm{He}$ concentration levels relevant for ITER.

This is illustrated in figure 10(left), where the heating efficiencies obtained in the experiments (symbols) are plotted as a function of the ${ }^{3} \mathrm{He}$ concentration in the plasma together with the theoretical predictions (lines). A distinct difference was observed between the electron and ion temperature responses to the modulated RF power (not shown here): while the $T_{\mathrm{e}}$ signal showed a small but clear response to the RF power changes at any ${ }^{3} \mathrm{He}$ concentration level, the ion temperature only started to be modulated at higher ${ }^{3} \mathrm{He}$ concentrations, suggesting that the ion absorption was increased in this regime. The ion response became stronger than the electron one when $\mathrm{X}\left[{ }^{3} \mathrm{He}\right]$ exceeded $20 \%$. This is consistent with the total heating efficiency changes from about $20 \%$ to $40 \%$ seen in figure 10 (left) when increasing the ${ }^{3} \mathrm{He}$ concentration from $5 \%$ to $25 \%$. This increase is seen to be solely due to the ion heating gradually becoming more efficient with increasing ${ }^{3} \mathrm{He}$ concentration, the electron heating essentially being unaffected. For the $N=2{ }^{3} \mathrm{He}$ heating scenario, the dependence of heating efficiency on the temperature was minor, at least in the limited temperature domain experimentally scanned. As in the $\mathrm{H}$ majority heating scheme the theoretical response mimics the experimental data: the electron absorption is unaffected by the change in the minority concentration while the ion heating 

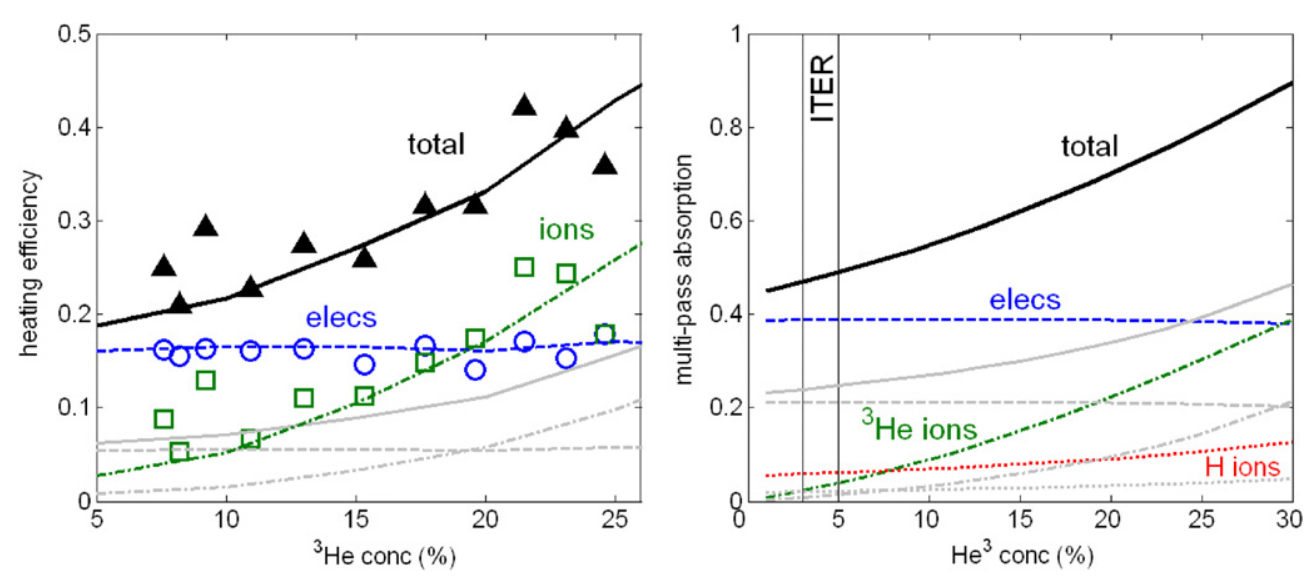

Figure 10. (left) Experimental heating efficiencies (ions-squares, electrons-circles, totaltriangles) obtained in the $N=2{ }^{3} \mathrm{He}$ heating experiments in JET (JPN 79352) as a function of the ${ }^{3} \mathrm{He}$ concentration together with the multi-pass absorption predictions (ions-dashed-dotted, electrons-dashed, total-solid) based on the single pass absorption values computed with the TOMCAT code (grey curves) considering $26 \%$ of power losses per wave pass in the plasma; (right) multi-pass absorption (ions-dashed-dotted, electrons-dashed, total-solid) estimated from the TOMCAT results for ITER's half-field plasma conditions adopting the same losses as found from the JET experiments (the grey curves indicate the single pass absorption values used in the multipass model).

gradually improves, a characteristic that is typical of second harmonic heating. In agreement with the experiments, numerical absorption estimates show that the electron and ion heating are equally important when $\mathrm{X}\left[{ }^{3} \mathrm{He}\right] \approx 20 \%$. To align the single pass absorption (grey curves) with the experimental heating efficiency (symbols), a 26\% single pass loss was assumed in the multi-pass absorption model, a somewhat higher value than that obtained for the $\mathrm{H}$ majority ICRH case. This is consistent with the higher fraction of RF-induced radiation losses observed in the $N=2{ }^{3} \mathrm{He}$ ICRH experiments compared with the $N=1 \mathrm{H}$ majority heating case in spite of the similar heating efficiencies: a $4 \mathrm{MW}$ modulation in power yields a $2 \mathrm{MW}$ amplitude response of the radiated power, i.e. about half of the coupled RF power is never used to heat the plasma but is lost through enhancement of the radiation processes.

When running the simulations for this second heating scheme for the half-field ITER parameters (see figure 10, right), it can be observed that qualitatively the same trend as observed in JET is to be expected, and that the single pass absorption (grey curves) will be modest $(\sim 25 \%)$ at the low ${ }^{3} \mathrm{He}$ concentrations being considered for ITER. Although this scheme would become much more efficient at higher ${ }^{3} \mathrm{He}$ concentrations, the amounts of ${ }^{3} \mathrm{He}$ that would be required for such an operation are prohibitive. Hence-similar to the previously discussed scheme - a projected heating efficiency of 50\% is foreseen for the ITER baseline conditions when adopting similar losses as those found for the JET experiments in the multipass absorption model.

Despite the low efficiency of this heating scenario, fast ${ }^{3} \mathrm{He}$ ions up to $200 \mathrm{keV}$ were detected by the neutral particle analyser (NPA) diagnostics when $5 \mathrm{MW}$ of RF power was applied. Although $N=2{ }^{3} \mathrm{He}$ heating was the intended scheme, a fraction of the launched power was also absorbed by fast D ions coming from NBI at their third harmonic ion-cyclotron resonance layer, which lies in between the antenna and the centrally located $N=2{ }^{3} \mathrm{He}$ ioncyclotron resonance (see figure $8(b)$ ). Since hydrogen beams were not available, D beams were used both for preheating the plasma and for obtaining the ion temperature from the 

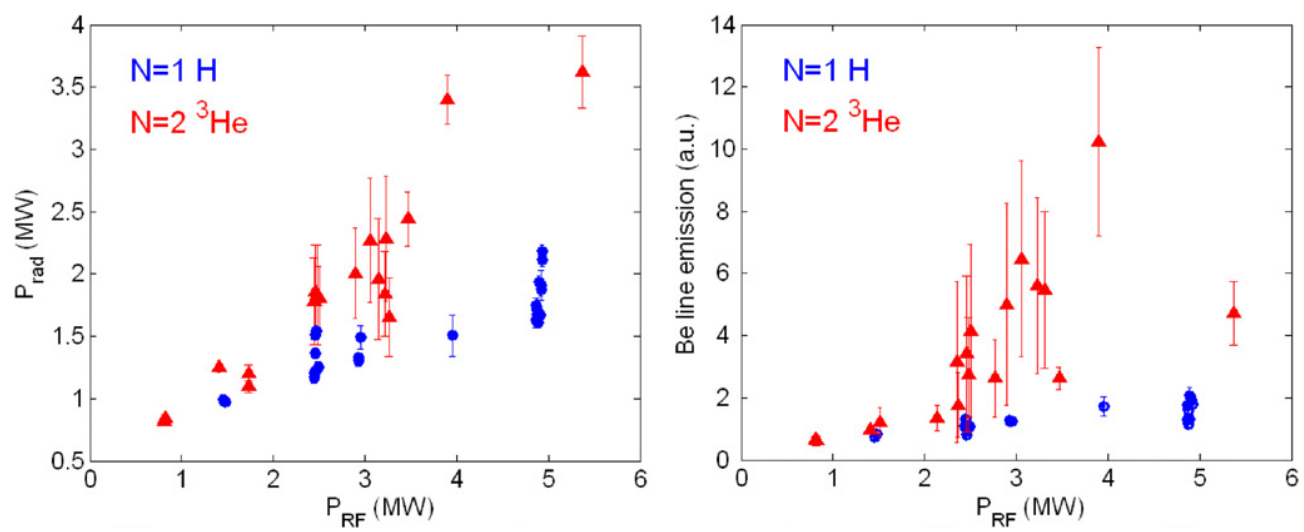

Figure 11. Total radiated power (left) and intensity of Be line (right) as a function of the ICRF power in a series of discharges of the $N=1 \mathrm{H}$ majority (circles) and the $N=2{ }^{3} \mathrm{He}$ (triangles) ICRF heating experiments (JPN 79330-79335, JPN 79343-79352).

charge exchange diagnostic. There was evidence that these particles were absorbing a nonnegligible part of the RF power: the fast ions detected by the NPA have energies that are a factor 2-3 times beyond the beam source energy $(80-130 \mathrm{keV})$ and the fast ion losses (measured with a scintillator probe) were strongly enhanced when D beams and ICRF were simultaneously applied to the plasma [34]. Note that no evidence of RF heated D particles was found in the fundamental $\mathrm{H}$ majority heating experiments although the 2 nd harmonic $\mathrm{D}$ cyclotron resonance lies in the plasma centre at the same location as the $N=1 \mathrm{H}$ cyclotron resonance (see figure $8(a)$ ). This illustrates the delicate interplay between these two auxiliary heating methods.

As briefly mentioned, an important consequence of the low ICRF absorptivity of these heating scenarios is the enhancement of plasma-wall interactions leading to relatively large radiation losses. This is depicted in figure 11(left), where the total radiated power is shown as a function of the ICRF power applied for the $N=1 \mathrm{H}$ (circles) and for the $N=2{ }^{3} \mathrm{He}$ (triangles) heating experiments. The data correspond to $0.4 \mathrm{~s}$ time averaged values sampled throughout the pulses. The density, temperature and NBI power $(\sim 1.3 \mathrm{MW})$ were similar in all the time intervals considered.

The fact that the radiation losses for a given ICRF power level are higher for the $N=2$ ${ }^{3} \mathrm{He}$ case than for the fundamental $\mathrm{H}$ majority case is not only due to the presence of relatively large fractions of ${ }^{3} \mathrm{He}$ in the plasma (higher $Z_{\text {eff }}$ ), but is also related to a stronger RF-induced plasma-wall interaction observed in the former case, leading to a higher impurity content in the plasma. This is depicted in figure 11(right), which shows the line emission intensity of beryllium (material of the ICRF antenna screen bars) measured by visible spectroscopy as a function of the ICRF power for the two scenarios. In this figure one can clearly see a significantly higher Be emission in the $N=2{ }^{3} \mathrm{He}$ case. The same time intervals as on the left figure were considered. A similar study for the $\mathrm{C}^{6+}$ and $\mathrm{C}^{4+}$ spectroscopy measurements (not shown) supported by $2 \mathrm{D}$ bolometer tomography indicates that most of the additional radiation observed in the $N=2{ }^{3} \mathrm{He}$ case comes from the plasma edge and the divertor region rather than from the bulk plasma.

Unlike for the non-active operation phase of ITER with ${ }^{4} \mathrm{He}$ plasmas, in which the wellestablished fundamental H minority ICRH scheme will be used (see [35] for its expected performance in ITER), further work is still necessary to identify the most optimal plasma 
parameters needed to exploit the hydrogen half-field ITER phase to its fullest. Apart from optimizing the above-described heating scenarios, one particularly promising heating scheme would be possible for $\mathrm{H}$ plasmas: by further reducing the confining magnetic field to $\sim 2 \mathrm{~T}$, the bulk hydrogen plasmas could be heated using second harmonic H ICRF heating. While such a reduced field may be too small to ensure good confinement, theoretical predictions suggest that it may be ideal for testing the RF system in ITER. Indeed, the expected single pass absorption for this scenario is nearly $100 \%$ with dominant ion heating [36], thanks to the enhancement of $N=2$ ion-cyclotron absorption with the resonant ion species concentration.

Finally, prior to actually using the fusion fuels $\mathrm{D}$ and $\mathrm{T}$, initial exploration of the performance of the ITER machine at full field $\left(B_{0}=5.3 \mathrm{~T}\right)$ will be done. In that case the ICRF heating schemes rely on heating ${ }^{3} \mathrm{He}$ ions at their fundamental cyclotron resonance layer in either $\mathrm{H}$ or ${ }^{4} \mathrm{He}$ plasmas (see figure $8(c)$ ). If ${ }^{3} \mathrm{He}$ minority heating in ${ }^{4} \mathrm{He}$ plasmas is a well-known and efficient heating scheme [37,38], ${ }^{3} \mathrm{He}$ heating in $\mathrm{H}$ plasmas is a more delicate scenario. For this scheme, referred to as an inverted heating scenario, the charge-to-mass ratio $(Z / A)$ of the minority is smaller than that of the majority and thus the mode-conversion layer at the ion-ion hybrid resonance is located at the low field side rather than at the high field side of the minority cyclotron resonance. This scenario was studied a few years ago at very low ${ }^{3} \mathrm{He}$ concentrations [39] and was recently revisited going to higher $\mathrm{X}\left[{ }^{3} \mathrm{He}\right]$ range [40]. Efficient ${ }^{3} \mathrm{He}$ minority heating is achieved only at very low concentrations $\mathrm{X}\left[{ }^{3} \mathrm{He}\right] \sim 1-2 \%$ (compared with $\sim 7-10 \%$ in standard non-inverted scenarios) with the mode-conversion regime already reached from $\sim 2 \%$ (whereas it is typically found around $12-15 \%$ in usual mode-conversion heating schemes). Heating efficiencies up to $70 \%$ are achieved at $\mathrm{X}\left[{ }^{3} \mathrm{He}\right] \sim 1 \%$ but the scenario is very sensitive to the gas mixture. Impurities may render it difficult to tune the parameters to ensure optimal heating: earlier as well as recent JET experiments identified the touchiness of the scheme to small amounts of D-like impurities (e.g. $\mathrm{C}^{6+}$ ) that come from the JET walls and contaminate the plasma. Other impurities such as $\mathrm{Be}^{4+}$ may play a similar role in ITER. However, provided the plasma composition can be carefully monitored and controlled, this scheme offers good potential for heating the ITER plasmas during its non-active full magnetic field operation phase.

\section{Summary and future}

During recent years, the JET experimental programme addressed a number of points that needed to be understood to guarantee successful operation of ICRF heating in next-step fusion devices such as ITER. Somewhat artificially subdividing the fate of RF power from the generators to the plasma particles into three coupled domains of interest, (1) generator-to-antenna, (2) antenna-to-edge-plasma and (3) edge-to-core-plasma, a series of achievements are presented in this paper.

(1) Power evolution between the generator and the antenna:

- Significant amounts of ICRF power ( $\sim 8 \mathrm{MW})$ have successfully been coupled to ELMy H-mode plasmas relying on optimized matching circuits between the antenna generator and the wave launchers. ELM resilience was demonstrated using both $3 \mathrm{~dB}$ hybrid couplers and conjugate-T configurations.

- ITER-relevant antenna voltages $(\sim 45 \mathrm{kV})$ and surface power densities $\left(\sim 6 \mathrm{MW} \mathrm{m}^{-2}\right)$ have been reached with the compact ILA without excessive increase in RF sheath effects and RF-induced plasma-wall interaction.

- Through combined inter-shot analysis and dedicated real-time matching experiments it was demonstrated that compact antenna arrays-although characterized by strong 
inter-strap cross-talk - can be successfully tuned to couple power to plasmas in a wide range of plasma conditions.

(2) Power coupled from the antenna to the plasma:

- Predictive and interpretative modelling tools were developed to allow further optimization of the design of ICRF antennas. A high degree of confidence in such tools was reached by cross-checking the theoretical calculations with the experimentally obtained values.

- Coupling computations done for some JET experiments have demonstrated the strong impact of the uncertainties of the SOL density profile on the calculations of the coupled power. Although the ICRF power specifications for ITER ( $P_{\mathrm{RF}}>20 \mathrm{MW}$ per antenna) are met under the most pessimistic density profiles considered, this is clearly an area to which more attention needs to be devoted in the future as the coupled power can differ up to an order of magnitude depending on the density profile facing the antenna.

- To guarantee optimal coupling, optimizing the antenna design is crucial. But since the exact shape and characteristics of the ITER SOL will not be known until the first plasmas are created in this machine, exploring methods for shaping the density profile in front of the antenna is a tool that can help in ensuring better coupling. Gas puff experiments done in JET and in other machines [24], only briefly mentioned here, showed promising results. The continuation of these studies planned for the near future should allow getting a firmer grip on the physics involved.

- There is clear evidence that non-absorbed RF power has a deleterious impact on the release of particles from plasma facing components, in particular in low single pass absorption schemes. Additionally, effects such as RF sheath-induced heat loads, not discussed here, require further attention. With the change of JET's first wall material from $\mathrm{C}$ to Be and tungsten $(\mathrm{W})$ [41], particular attention will be given to this research area with experiments to be conducted in the near future to assess the interaction between ICRF power and such metallic walls.

(3) Power absorption inside the plasma:

- ICRF heating schemes for ITER's activated phase were already studied in JET two decades ago (see [42] and references therein). New experiments are planned to take place in the following years in the presence of the Be wall and $\mathrm{W}$ divertor [43]. However, no major obstacles are expected for operating the ICRF system in ITER's activated DT phase.

- Both $\mathrm{H}$ and ${ }^{4} \mathrm{He}$ are foreseen as the main working gases in ITER's non-activated phase, when the machine will be operated at half its nominal magnetic field $\left(B_{0}=2.65 \mathrm{~T}\right)$. Heating $\mathrm{H}$ ions at their fundamental cyclotron frequency in ${ }^{4} \mathrm{He}$ plasmas is a wellestablished heating scheme and is expected to perform well in ITER. Numerical simulations indicate a heating efficiency of $80-90 \%$ for this scheme under the reference half-field plasma conditions if the $\mathrm{H}$ concentration is kept below $20 \%$ [35]. For $\mathrm{H}$ plasmas two heating schemes are available: fundamental majority $\mathrm{H}$ and second harmonic ${ }^{3} \mathrm{He}$ ICRF heating. Both these schemes were tested recently in JET, adopting the exact ITER magnetic field, RF working frequency and electron density but evidently operating at a lower temperature. While neither of the two is overly promising, they offer sufficient potential for application in ITER within their moderate heating capabilities.

- The results from the fundamental H majority ICRF experiments confirmed the low heating efficiency expected from theory (the wave polarization near the minority 
cyclotron layer prohibits efficient ion absorption). Simplified numerical predictions for ITER's half-field plasma parameters suggest a moderate heating efficiency ( $\sim 50 \%$ ) with margin for performance optimization based on the dilution of the $\mathrm{H}$ plasmas with another ion species (e.g. ${ }^{4} \mathrm{He}$ ) to change the wave polarization or by operation at higher plasma temperatures to broaden the Doppler-shifted ICRF absorption.

- Second harmonic ${ }^{3} \mathrm{He}$ cyclotron heating equally proved to be of limited potential in JET. Although this heating scheme becomes more efficient at high ${ }^{3} \mathrm{He}$ concentrations, it is characterized by low single pass absorption when typical ${ }^{3} \mathrm{He}$ concentrations expected for ITER are considered. The heating efficiency estimated for ITER is also around $50 \%$ but in contrast to the results of $\mathrm{H}$ majority heating scheme, this value is not very sensitive to the bulk plasma temperature.

- Experiments on fundamental ICRF heating of ${ }^{3} \mathrm{He}$ minority ions in $\mathrm{H}$ plasmas, a heating scheme to be used in the non-active full-field operation phase of ITER, have shown that this is a well-performing scenario if the ${ }^{3} \mathrm{He}$ concentration is kept below 1-2\%. The strong influence of impurities such as $\mathrm{C}$ or Be on the heating efficiency and on the transition to the mode-conversion heating regime has been confirmed.

In conclusion it can be stated that significant progress has been made over the last years to make ICRF heating a robust heating scheme for the next-step fusion devices. From the antenna tuning, coupling as well as heating points of view, RF heating is getting ready for ITER, with a number of issues-e.g. a better understanding of the interaction between the RF waves and the plasma edge and first wall- to be further addressed in the coming years.

\section{Acknowledgments}

This work was supported by EURATOM and carried out within the framework of the European Fusion Development Agreement. The views and opinions expressed herein do not necessarily reflect those of the European Commission.

Euratom (C) 2011.

\section{References}

[1] ITER Team et al 1999 Nucl. Fusion 392137

[2] Lamalle P et al 2009 18th Topical Conf. on RF Power in Plasmas (Gent, Belgium), AIP Conf. Proc. 1187265

[3] Kaye A et al 1994 Fusion Eng. Des. 74 1-21

[4] Mayoral M-L et al 2007 17th Topical Conf. on RF Power in Plasmas (Clearwater, USA), AIP Conf. Proc. 933 143

[5] Noterdaeme J-M et al 1999 13th Topical Conf. on RF Power in Plasmas (Annapolis, IN), AIP Conf. Proc. 48592

[6] Pozar D 2005 Microwave Engineering 3rd edn (New York: Wiley)

[7] Monakhov I et al 2009 18th Topical Conf. on RF Power in Plasmas (Gent, Belgium), AIP Conf. Proc. 1187205

[8] Mayoral et al 2009 36th EPS Conf. on Plasma Physics (Sofia, Bulgaria) vol 33E (ECA) p O-4.048

[9] Durodie F et al 2005 Fusion Eng. Des. $74223-8$

[10] Nightingale M et al 2009 18th Topical Conf. on RF Power in Plasmas (Gent, Belgium), AIP Conf. Proc. 1187 213

[11] V P Bhatnagar et al 1989 Plasma Phys. Control. Fusion 31211

[12] Litwin C 1988 Phys. Rev. Lett. $602375-8$

[13] Durodie F et al 2010 23rd IAEA Fusion Energy Conf. 2010 (Daejeon, Korea)

[14] Bilato R et al 2005 Nucl. Fusion 45 L5-L7

[15] Milanesio D and Maggiora R 2010 Nucl. Fusion 50025007

[16] Messiaen A and Weynants R 2011 Plasma Phys. Control. Fusion 53 1-29

[17] Messiaen A et al 2010 Nucl. Fusion 50 1-21

[18] Lerche E et al 2009 18th Conf. on RF Power in Plasmas (Gent, Belgium), AIP Conf. Proc. 118793 
[19] Van Eester D et al 1998 Plasma Phys. Control. Fusion 40 1949-75

[20] Jacquet Ph et al 2011 Nucl. Fusion 51103018

[21] Czarnecka A et al 2011 Plasma Phys. Control. Fusion submitted (special TF-H edition)

[22] Perkins F W et al 1989 Nucl. Fusion 29 583-92

[23] Bobkov V et al 2009 18th Topical Conf. on RF Power in Plasmas (Gent, Belgium), AIP Conf. Proc. 1187125

[24] Mayoral M L et al 23rd IAEA Fusion Energy Conf. 2010 (Daejeon, Korea)

[25] Wassenhove G et al 2011 38th EPS Conf. on Plasma Physics (Strasbourg, France)

[26] Bergeaud V et al 2000 Nucl. Fusion 4035

[27] Van Eester D et al 2002 Nucl. Fusion 42 310-28

[28] Perkins F W et al 1977 Nucl. Fusion 171197

[29] Lerche E et al 2010 37th EPS Conf. on Plasma Physics (Dublin, Ireland) vol 34A (ECA) O4.121

[30] Lerche E et al 2008 Plasma Phys. Control. Fusion 50035003

[31] von Hellermann M G et al 1990 Rev. Sci. Instrum. 613479

[32] de la Luna E et al 2004 Rev. Sci. Instrum. 753831

[33] Van Eester D et al 2009 Plasma Phys. Control. Fusion 51044007

[34] Kiptily V et al 2012 Fast ions in mode conversion heating $\left({ }^{3} \mathrm{He}\right)-\mathrm{H}$ plasmas in JET Plasma Phys. Control. Fusion (special TF-H edition)

[35] Lerche E et al 2011 19th Topical Conf. on RF Power in Plasmas (Newport, USA)

[36] Lerche E et al 23rd IAEA Fusion Energy Conf. (Daejeon, Korea)

[37] Mantsinen et al 2004 Nucl. Fusion 44 33-46

[38] Mantsinen et al 2002 Phys. Rev. Lett. 89

[39] Mayoral M-L et al 2006 Nucl. Fusion 46 S-550-63

[40] Van Eester D et al 2012 Heating $\left({ }^{3} \mathrm{He}\right)-\mathrm{H}$ JET plasmas with multiple mode conversion layers Plasma Phys. Control. Fusion (special TF-H edition)

[41] Neu R et al 2011 38th EPS Conf. on Plasma Physics (Strasbourg, France)

[42] Start D et al 1999 Nucl. Fusion 39321

[43] Sips A C C et al 2011 38th EPS Conf. on Plasma Physics (Strasbourg, France) 